\title{
Maximum key-profile correlation (MKC) as a measure of tonal structure in music
}

\author{
ANNIE H. TAKEUCHI \\ Johns Hopkins University, Baltimore, Maryland
}

\begin{abstract}
Tonal structure is musical organization on the basis of pitch, in which pitches vary in importance and rate of occurrence according to their relationship to a tonal center. Experiment 1 evaluated the maximum key-profile correlation (MKC), a product of Krumhansl and Schmuckler's key-finding algorithm (Krumhansl, 1990), as a measure of tonal structure. The MKC is the maximum correlation coefficient between the pitch class distribution in a musical sample and key profiles, which indicate the stability of pitches with respect to particular tonal centers. The MKC values of melodies correlated strongly with listeners' ratings of tonal structure. To measure the influence of the temporal order of pitches on perceived tonal structure, three measures (fifth span, semitone span, and pitch contour) taken from previous studies of melody perception were also correlated with tonal structure ratings. None of the temporal measures correlated as strongly or as consistently with tonal structure ratings as did the $\mathrm{MKC}$, and nor did combining them with the MKC improve prediction of tonal structure ratings. In Experiment 2, the MKC did not correlate with recognition memory of melodies. However, melodies with very low MKC values were recognized less accurately than melodies with very high MKC values. Although it does not incorporate temporal, rhythmic, or harmonic factors that may influence perceived tonal structure, the MKC can be interpreted as a measure of tonal structure, at least for brief melodies.
\end{abstract}

Music can be organized on the basis of pitch, temporal pattern, loudness, and timbre, or on combinations of these dimensions. Pitch is particularly important in music, and a major aspect of pitch organization is tonality or tonal structure. Tonal structure consists of two types of regularities. The first is key membership. In melodies with high degrees of tonal structure, pitches are generally drawn from a single key, that is, they correspond to the pitches of a single major or minor scale. Tonal structure also requires the functional differentiation of pitches. Depending on their relationship to the tonal center, certain pitches are more stable than others, and in tonal melodies, more stable pitches occur more frequently and act as points of departure and arrival for less stable pitches, which occur less frequently (Krumhansl, 1990). In atonal ${ }^{1}$ melodies, there is no tonal center and pitches are undifferentiated with respect to either function or rate of occurrence.

Several studies have demonstrated the effect of tonal structure on the perceptual processing of musical pat-

\footnotetext{
This article is part of a $\mathrm{PhD}$ dissertation in psychology at Johns Hopkins University. Data were presented, in part, at the Second International Conference on Music Perception and Cognition, Los Angeles (February 1992). This research was supported by a National Science Foundation predoctoral grant. Many thanks are extended to Stewart $\mathbf{H}$. Hulse and Louis D. Braida for providing support and advice for this research and comments on the manuscript, and to Annabel Cohen and Richard Parncutt for their comments and discussion of this work. Correspondence concerning this article should be addressed to $\mathrm{A}$. $\mathrm{H}$. Takeuchi, National Analysts, Inc., 1700 Market St., Philadelphia, PA 19103-3913.
}

terns. Tonal melodies are better remembered than atonal melodies (Cohen, 1982; Dewar, Cuddy, \& Mewhort, 1977; Frances, 1958/1988; Watkins, 1985b). Individual pitches within melodies are better remembered when the melodies are tonal rather than atonal (Dewar et al.,1977; Krumhansl, 1979; Long, 1977). It is also easier to detect a mistuned note in a tonal than in an atonal context (Lynch, Eilers, Oller, Urbano, \& Wilson, 1991; Umemoto, Mikumo, \& Murase, 1989).

In some studies of the effects of tonal structure (e.g., Dewar et al., 1977; Umemoto et al., 1989), tonal structure has been operationalized as a dichotomy. A tonal melody was one in which all of the pitches were drawn from a single key, and atonal melodies consisted of random pitches. However, tonal structure is more appropriately viewed as a matter of degree than as a dichotomy. Melodies vary in the number and proportion of nonscale pitches. The degree to which pitches are functionally differentiated also cannot be fully expressed in terms of a dichotomy.

Moreover, listeners perceive degrees of tonal structure. Cuddy and her colleagues (Cuddy, Cohen, \& Mewhort, 1981; Cuddy \& Lyons, 1981) described five levels of tonal structure and composed melodies at each of these levels. The levels were distinguished by the number of nonscale pitches in the melodies, the implied harmonic structure of the melodies, and the selection of scale pitches. Listeners' ratings of the tonal structure of the melodies decreased monotonically with the level of tonal structure. Taylor (1976) determined the tonal strength of melodies by measuring the consistency with 
which different listeners sang the same pitch when asked to sing the tonal center of a melody. The different tonal strength values he obtained for different melodies also suggests that listeners perceive degrees of tonal structure.

In view of listeners' sensitivity to degrees of tonal structure, a nondichotomous measure should better describe perceived tonal structure, and could also produce more precise predictions of the effects of tonal structure on the perception and memory of musical patterns than can a dichotomous measure. Such a measure of tonal structure should not require the user to make empirical measurements, as Taylor's (1976) measure of tonal strength described above does. Also, it should be applicable to musical samples of any length, and with any number of tones simultaneously presented.

The maximum key-profile correlation (MKC), a product of Krumhansl and Schmuckler's key-finding algorithm (Krumhansl, 1990), meets these criteria. The MKC does not require the user to make empirical measurements and is applicable to musical samples of any length and texture. The MKC is likely to be a good measure of perceived tonal structure because its input is the distribution of pitch classes in a musical sample, and this distribution indicates the extent of both key membership and functional differentiation, which are two important determinants of tonal structure, as discussed above.

The MKC is a product of an algorithm for determining the key of a musical sample. However, in the current application, the central question is not what key a musical sample is in, but to what degree a musical sample fits the key, or more generally, the degree of tonal structure of the sample. This distinction between the key of a musical sample and its degree of tonal structure is an important one. The key of a musical sample is not of particular concern to a listener, because pitch structure in music depends on relations among pitches and not on absolute pitches. Thus, the key of a musical sample, that is, the absolute pitch of the tonal center, is of little relevance to a listener's perception of music. However, the degree of tonal structure is relevant to a listener. It influences a listener's perception and memory of melodies and of notes within melodies as described above, and very likely also affects perception and memory of larger musical units.

The key-finding algorithm (Krumhansl, 1990) is based on key profiles which represent the stability of each pitch class within the context of a particular musical key. Pitch class refers to the letter name of a pitch, regardless of its octave. Krumhansl and Kessler (1982) derived numerical values for key profiles using a probe-tone rating task. In this task, listeners heard a musical pattern that established a particular musical key. They then rated, on a scale of 1 to 7 , how well various probe tones that varied only in pitch fit into the tonal context established by the pattern, with higher ratings indicating better fit. The averaged ratings provided numerical values for the key profiles used in the key-finding algorithm. The psychological validity of the key profiles has been confirmed in several experiments (Frankland \& Cohen, 1990;
Janata \& Reisberg, 1988; Krumhansl, 1979; Krumhansl \& Kessler, 1982; Krumhansl \& Shepard, 1979).

Because key profiles depend only on the relationship between a pitch and the tonal center and not on absolute pitches, the key profiles for the major keys are all transpositions of each other, as are the key profiles for minor keys. For example, the values of the key profile for $\mathrm{C}$ major can be shifted up by one position to produce the values of the key profile for $\mathrm{C}$ major, because the stability of the pitch $\mathrm{C}$ in the key of $\mathrm{C}$ major is equivalent to the stability of the pitch $\mathrm{C} \#$ in the key of $\mathrm{C}$ major. This transposability of key profiles was validated by Krumhansl and Kessler (1982), who found that probe-tone ratings for tones presented in different major and different minor key contexts were virtually transpositions of each other. The values for the major- and minor-key profiles were published in Krumhansl (1990).

The key-finding algorithm is based on the assumption that the most stable pitch classes should occur most often. It therefore finds the maximum correlation between the distribution of pitch classes in a musical sample and the values of a key profile for a particular key. The distribution of pitch classes is represented by the input vector, a set of 12 numbers indicating the total duration in the sample of each pitch class from $C$ to $B$. The input vector is correlated with each of 24 key profiles, one for each major and minor key. The key profile that produces the maximum correlation coefficient with the input vector indicates the most likely key of the musical sample. The value of the maximum correlation coefficient, which is the MKC, is the measure whose interpretation as a measure of degree of tonal structure is evaluated in Experiment 1. A higher MKC value should indicate a greater degree of tonal structure.

This interpretation of the MKC as a measure of the degree of tonal structure is implicit in Krumhansl's (1990) description and tests of the key-finding algorithm. Parncutt (1989) has also interpreted the MKC as an indicator of the clarity of tonal structure. However, this interpretation of the MKC has not been directly tested. Therefore, in Experiment 1, the MKC values of melodies were correlated with subjects' ratings of the tonal structure of brief melodies. Ratings and other measures of perceived tonal structure from several other studies were also correlated with the MKC.

A weakness of the MKC is that it does not take into account the temporal order in which tones are presented; the MKC is based solely on the distribution of pitch classes. Temporally reordering a set of pitches can change the perceived tonal center (Brown, 1987, 1988; Butler, 1990; Butler \& Brown, 1984). It is therefore likely that temporal reordering can also change the perceived degree of tonal structure. Three measures that reflect the temporal ordering of tones were therefore correlated, both independently and in combination with the MKC, with ratings of tonal structure in order to determine whether they improved the prediction of tonal-structure ratings. 
Experiment 2 evaluated whether the general finding that more tonal melodies were more easily remembered than less tonal melodies could be refined by using the MKC as a measure of tonal structure. Specifically, the experiment tested the prediction that memory performance on individual melodies would correlate with the MKC values of the melodies.

\section{EXPERIMENT 1}

Experiment 1 evaluated the $\mathrm{MKC}$ as a measure of tonal structure by correlating the MKC values of novel melodies with listeners' ratings of tonal structure. Also, the ratings made by musically trained and untrained listeners were compared to determine whether they perceived tonal structure similarly. Musically trained listeners might differentiate degrees of tonal structure more finely than untrained listeners because of their greater knowledge of music. Alternatively, listeners' perceptions of tonal structure may not depend on specific musical knowledge, in which case musical training would have no effect on tonal structure ratings.

The MKC was also compared with a similar measure based only on simplified key profiles which weighted all scale tones equally. Comparison of this measure with the MKC evaluated the need for differentially weighting the scale tones. Finally, three measures reflecting the temporal ordering of tones in melodies were compared with the MKC, and were also combined with the MKC in multiple regression analyses to determine whether they improved the prediction of tonal structure ratings.

\section{Method}

\section{Subjects}

Thirty members of the Johns Hopkins University community participated for pay or as a course requirement. Fifteen subjects were musicians who had had between 4 and 12 years of private music lessons. The other 15 subjects were nonmusicians who had had less than 2 years of private music lessons and did not currently play any musical instruments.

\section{Stimuli}

Thirty-six seven-note melodies with MKC values ranging from 0.35 to 0.94 were constructed. Twelve of these melodies were produced by randomly selecting pitches, with the restriction that consecutive repeating pitches were not allowed. The remaining melodies were produced by changing or reordering pitches to increase the MKC values of the melodies. Twelve melodies were in minor keys, and 24 were in major keys, according to the key-finding algorithm. The melodies ranged in pitch from $G_{3}(196.0 \mathrm{~Hz})$ to $G_{5}$ $(784.0 \mathrm{~Hz})$ and are presented in Table 1.

In addition, 12 practice melodies were composed. These melodies ranged in MKC values from 0.28 to 0.94 . Each of the practice melodies was in a different major or minor key according to the key-finding algorithm, with 3 of the melodies in minor keys and 9 in major keys. The practice melodies ranged in pitch from $\mathrm{A}_{3}(220.0 \mathrm{~Hz})$ to $\mathrm{D} \#_{5}(622.3 \mathrm{~Hz})$.

Melodies were presented at a rate of 4 notes $/ \mathrm{sec}$, and each note was $250 \mathrm{msec}$ in duration. The duration of each melody was therefore $1.75 \mathrm{sec}$. Tones were produced by a Yamaha DX7-IIFD synthesizer controlled by an IBM-AT clone computer via a Roland
MPU-401 musical instrument digital interface. The synthesizer produced additive synthesis of six tone generators that had identical amplitude envelopes and frequencies that were the fundamental frequency and the first five overtones. The melodies were recorded monophonically on a Sony TC-R503 cassette tape at 3.75 ips. The tape recorder was connected to a Crown D-75 amplifier and to a single Auratone T-6 monitor for playback. The cassette tape was played to subjects at a comfortable listening level.

\section{Procedure}

The subjects were instructed to rate how tonal or atonal each melody was on a scale of 1 to 7. A rating of 1 indicated that the melody was very atonal, and 7 indicated the melody was very tonal. Instructions stated that atonal melodies sounded like random sets of notes and did not seem to be in any key. Tonal melodies made sense as a tune, and the notes were organized in a key. The subjects were also informed that there were no correct or incorrect answers for the task; they were to make subjective judgments about the melodies. Each trial began with an announcement of the trial number, followed $1.5 \mathrm{sec}$ later by one of the melodies. The subjects had $4.25 \mathrm{sec}$ to write their responses on answer sheets before the next trial number was announced.

To become familiarized with the range of tonal structure in the melodies, the subjects first completed a practice block in which they rated the 12 practice melodies. These ratings were not included in the data analysis. The subjects then rated each of the 36 melodies three times in four blocks of 27 trials each. The melodies were presented in random order without replacement.

The subjects were tested in a quiet room in groups of up to 4 . After completing all trials, which took about $20 \mathrm{~min}$, the subjects answered a short questionnaire about their musical experience.

\section{Results}

\section{Analysis of Ratings}

To normalize differences in the use of the rating scale across subjects, each subject's ratings were converted to $z$ scores. Positive $z$ scores indicate high ratings of tonal structure; negative $z$ scores indicate low ratings of tonal structure.

\section{Cluster Analysis}

To group subjects who rated the melodies similarly, a hierarchical cluster analysis was performed. The analysis used the cluster procedure in SAS (Cary, NC) with the centroid method (Sokal \& Michener, 1958). To determine a distance measure among subjects, correlations were computed between each subject's average rating of each melody and every other subject's average ratings of the same melodies. Each value in the resultant correlation matrix was subtracted from 1 to derive a distance matrix.

The analysis revealed three clusters of subjects and 3 "unusual" subjects. Cluster A consisted of 12 musicians, with an average of 7.8 years of private music lessons $(S D=2.7)$, and 4 nonmusicians. Cluster $B$ consisted of 2 musicians, each of whom reported 5 years of private music lessons, and 5 nonmusicians. Cluster $\mathrm{C}$ consisted of 1 musician, with 5 years of private music lessons, and 3 nonmusicians. All 3 unusual subjects were nonmusicians. Musicians were more likely than nonmusicians to be in Cluster $\mathrm{A}\left[\chi^{2}(1, N=30)=6.57^{*}\right] .^{2}$ 
Table 1

Melodies, Ratings from Experiment 1, and Error Percentages from Experiment 2

\begin{tabular}{|c|c|c|c|c|c|c|c|c|c|c|c|c|c|}
\hline \multirow[b]{2}{*}{ Melody* } & \multirow[b]{2}{*}{$\mathrm{MKC}$} & \multirow{2}{*}{$\begin{array}{l}\text { Simplified } \\
\text { MKC }\end{array}$} & \multirow{2}{*}{$\begin{array}{c}5 \text { th } \\
\text { Span } \\
\end{array}$} & \multirow{2}{*}{$\begin{array}{l}\% 5 \text { th } \\
\text { Span }\end{array}$} & \multirow{2}{*}{$\begin{array}{c}\text { Semitone } \\
\text { Span }\end{array}$} & \multirow{2}{*}{$\begin{array}{c}\text { \%Semitone } \\
\text { Span }\end{array}$} & \multirow[b]{2}{*}{ Contour } & \multirow[b]{2}{*}{$\%$ Contour } & \multicolumn{3}{|c|}{$\begin{array}{c}\text { Experiment } \mathrm{I} \\
\text { Cluster }\end{array}$} & \multicolumn{2}{|c|}{$\begin{array}{c}\text { Experiment } 2 \\
\text { Cluster }\end{array}$} \\
\hline & & & & & & & & & A & $\mathrm{B}$ & $\mathrm{C}$ & Large & Small \\
\hline $\mathrm{D}_{5} \mathrm{C}_{5}\left(\mathrm{CH}_{5}\right) \mathrm{A}_{4} \mathrm{~B}_{4} \mathrm{~A}_{4} \mathrm{~F}_{4} \mathrm{G}_{4}$ & 0.35 & 0.31 & 0 & 0 & 0 & 0 & 1 & 50 & -0.60 & 0.08 & -0.24 & 72 & 38 \\
\hline $\mathrm{C}_{4} \mathrm{Ft}_{4} \mathrm{G}_{4}\left(\mathrm{G}_{4}\right) \mathrm{F}_{4} \mathrm{GH}_{4} \mathrm{Ft}_{4} \mathrm{~B}_{3}$ & 0.36 & 0.24 & 1 & 17 & 0 & 33 & 1 & 50 & -0.56 & 0.13 & -0.85 & 42 & 43 \\
\hline $\mathrm{B}_{4} \mathrm{DH}_{5} \mathrm{D}_{5} \mathrm{~F}_{4} \mathrm{~A}_{4}\left(\mathrm{C}_{5}\right) \mathrm{CH}_{5} \mathrm{E}_{4}$ & 0.36 & 0.31 & 1 & 17 & 0 & 33 & 1 & 50 & -0.81 & -0.92 & -0.36 & 12 & 26 \\
\hline$E_{5} G_{4}^{*} C_{5} F_{5}\left(G_{5}\right) A_{4} G_{4} A_{4}$ & 0.37 & 0.66 & 1 & 17 & 0 & 33 & 1 & 50 & -0.93 & -0.89 & -0.38 & 1 & 5 \\
\hline $\mathrm{Eb}_{4} \mathrm{~A}_{4} \mathrm{Db}_{5}\left(\mathrm{D}_{5}\right) \mathrm{B}_{4} \mathrm{Ab}_{4} \mathrm{~F}_{4} \mathrm{G}_{4}$ & 0.38 & 0.31 & 1 & 17 & 0 & 17 & 0 & 33 & -0.64 & -0.34 & -0.04 & 18 & 36 \\
\hline $\mathrm{F}_{4} \mathrm{CH}_{5} \mathrm{C}_{5} \mathrm{D}_{5}\left(\mathrm{E}_{5}\right) \mathrm{G}_{4} \mathrm{E}_{4} \mathrm{~A}_{4}$ & 0.38 & 0.31 & 1 & 33 & 1 & 50 & 1 & 67 & -0.72 & -0.47 & -0.20 & 4 & 14 \\
\hline $\mathrm{Ab}_{3} \mathrm{C}_{4} \mathrm{D}_{4}\left(\mathrm{E}_{4}\right) \mathrm{G}_{4} \mathrm{Db}_{4} \mathrm{~A}_{3} \mathrm{~B} b_{3}$ & 0.41 & 0.31 & 1 & 17 & 0 & 17 & 0 & 33 & -0.35 & 0.44 & -0.07 & 21 & 38 \\
\hline $\mathrm{Bb}_{3} \mathrm{~F}_{4} \mathrm{E}_{4} \mathrm{~A}_{3}\left(\mathrm{G}_{3}\right) \mathrm{Eb}_{4} \mathrm{Gr}_{4} \mathrm{C}_{4}$ & 0.41 & 0.66 & 1 & 33 & 1 & 67 & 1 & 50 & -0.66 & 0.02 & -0.33 & 4 & 14 \\
\hline $\mathrm{CH}_{4} \mathrm{G}_{4} \mathrm{CH}_{4} \mathrm{~F}_{4} \mathrm{~A}_{4} \mathrm{D}_{4}\left(\mathrm{DH}_{4}\right) \mathrm{C}_{4}$ & 0.42 & 0.51 & 1 & 50 & 1 & 50 & 1 & 50 & -0.65 & -0.35 & -0.11 & 16 & 31 \\
\hline $\mathrm{C}_{4} \mathrm{G}_{4} \mathrm{CH}_{4} \mathrm{~F}_{4} \mathrm{~A}_{4} \mathrm{C}_{4}\left(\mathrm{~A}_{3}\right) \mathrm{CH}_{4}$ & 0.52 & 0.38 & 1 & 67 & 1 & 50 & 1 & 67 & 0.30 & -0.05 & 0.30 & 4 & 36 \\
\hline $\mathrm{G}_{4} \mathrm{~A}_{4} \mathrm{C}_{5} \mathrm{~B}_{4} \mathrm{~F}_{5}\left(\mathrm{FH}_{5}\right) \mathrm{G}_{4} \mathrm{~F}_{4}$ & 0.57 & 0.51 & 1 & 17 & 0 & 33 & 1 & 50 & -0.78 & -0.88 & -0.59 & 8 & 21 \\
\hline$F_{4} \mathrm{CH}_{5} \mathrm{~B}_{4} \mathrm{D}_{5}\left(\mathrm{E}_{5}\right) \mathrm{G}_{4} \mathrm{~F}_{4} \mathrm{AH}_{4}$ & 0.59 & 0.66 & 1 & 17 & 0 & 33 & 1 & 67 & -0.63 & -0.57 & -0.38 & 1 & 19 \\
\hline $\mathrm{F}_{4} \mathrm{E}_{4}\left(\mathrm{~F}_{4}\right) \mathrm{B}_{3} \mathrm{~A}_{3} \mathrm{G}_{3} \mathrm{~A}_{3} \mathrm{C}_{4}$ & 0.60 & 0.66 & 1 & 33 & 0 & 0 & 0 & 17 & -0.18 & 0.27 & -0.39 & 19 & 38 \\
\hline $\mathrm{F}_{4} \mathrm{~A}_{4} \mathrm{C}_{5} \mathrm{~Eb}_{5} \mathrm{D}_{5}\left(\mathrm{D} \mathrm{b}_{5}\right) \mathrm{Bb}_{4} \mathrm{~A}_{4}$ & 0.62 & 0.77 & 0 & 0 & 0 & 0 & 0 & 17 & 0.61 & 0.22 & 0.79 & 13 & 36 \\
\hline $\mathrm{Ab}_{3} \mathrm{C}_{4} \mathrm{D}_{4}\left(\mathrm{D}_{4}\right) \mathrm{G}_{4} \mathrm{D}_{4} \mathrm{Ab}_{3} \mathrm{C}_{4}$ & 0.62 & 0.57 & 1 & 33 & 0 & 33 & 0 & 33 & -0.28 & 0.55 & -0.87 & 6 & 24 \\
\hline $\mathrm{B}_{3} \mathrm{FH}_{4} \mathrm{G}_{4}\left(\mathrm{G}_{4}\right) \mathrm{F}_{4} \mathrm{G}_{4} \mathrm{FH}_{4} \mathrm{~B}_{3}$ & 0.65 & 0.57 & 0 & 0 & 0 & 33 & 1 & 50 & 0.38 & -0.04 & -0.12 & 14 & 31 \\
\hline $\mathrm{B}_{4} \mathrm{D} \#_{5} \mathrm{CH}_{5} \mathrm{E}_{4} \mathrm{Al}_{4}\left(\mathrm{C}_{5}\right) \mathrm{C}_{5} \mathrm{E}_{4}$ & 0.67 & 0.65 & 1 & 17 & 1 & 50 & 1 & 50 & -0.73 & -0.43 & -0.14 & 5 & 19 \\
\hline $\mathrm{G}_{4} \mathrm{E}_{4}\left(\mathrm{~F}_{4}\right) \mathrm{B}_{3} \mathrm{~A}_{3} \mathrm{G}_{3} \mathrm{~A}_{3} \mathrm{C}_{4}$ & 0.67 & 0.65 & 0 & 0 & 0 & 0 & 0 & 17 & 0.54 & 0.94 & -0.15 & 19 & 38 \\
\hline $\mathrm{F}_{4} \mathrm{Ab}_{4} \mathrm{C}_{5} \mathrm{~Eb}_{5} \mathrm{Db}_{5}\left(\mathrm{~B}_{4}\right) \mathrm{Bb}_{4} \mathrm{~A}_{4}$ & 0.67 & 0.66 & 1 & 17 & 0 & 0 & 0 & 17 & -0.28 & -0.18 & 0.06 & 9 & 14 \\
\hline $\mathrm{D}_{5} \mathrm{Cl}_{5}\left(\mathrm{C}_{5}\right) \mathrm{A}_{4} \mathrm{~B}_{4} \mathrm{Al}_{4} \mathrm{Ft}_{4} \mathrm{~A}_{4}$ & 0.68 & 0.51 & 0 & 0 & 0 & 0 & 1 & 50 & -0.21 & -0.26 & 0.47 & 18 & 36 \\
\hline $\mathrm{E}_{5} \mathrm{G}_{4}^{\sharp_{4}} \mathrm{C}_{5} \mathrm{E}_{5}\left(\mathrm{~F}_{5}\right) \mathrm{A}_{4} \mathrm{Ft}_{4} \mathrm{~A}_{4}$ & 0.69 & 0.51 & 1 & 17 & 0 & 33 & 1 & 50 & -0.80 & -0.70 & -0.03 & 1 & 12 \\
\hline $\mathrm{Bb}_{3} \mathrm{~F}_{4} \mathrm{E}_{4} \mathrm{~A}_{3}\left(\mathrm{G}_{3}\right) \mathrm{Eb}_{4} \mathrm{G}_{4} \mathrm{~B}_{3}$ & 0.69 & 0.51 & 1 & 17 & 1 & 67 & 1 & 50 & -0.28 & 0.15 & -0.39 & 12 & 36 \\
\hline$D_{5} C \#_{5}\left(C_{5}\right) G_{4} B_{4} A_{4} F_{4} A_{4}$ & 0.76 & 0.77 & 1 & 17 & 0 & 17 & 1 & 50 & -0.37 & -0.50 & 0.46 & 10 & 41 \\
\hline $\mathrm{G}_{4} \mathrm{~A}_{4} \mathrm{C}_{5} \mathrm{~B}_{4} \mathrm{E}_{5}\left(\mathrm{DH}_{5}\right) \mathrm{F}_{4} \mathrm{G}_{4}$ & 0.78 & 0.77 & 0 & 0 & 0 & 17 & 1 & 67 & 0.58 & -0.59 & 0.00 & 16 & 19 \\
\hline$B_{4} E_{5} C_{5}^{*} E_{4} A_{4}\left(G_{4}^{*}\right) C E_{5} E_{4}$ & 0.78 & 0.52 & 0 & 0 & 0 & 33 & 1 & 50 & -0.06 & $-0.6 \mathrm{I}$ & -0.35 & 9 & 45 \\
\hline $\mathrm{Eb}_{4} \mathrm{Ab} b_{4} \mathrm{Db}_{5}\left(\mathrm{D}_{5}\right) \mathrm{B} b_{4} \mathrm{Ab} b_{4} \mathrm{~F}_{4} \mathrm{G}_{4}$ & 0.79 & 0.77 & 0 & 0 & 0 & 0 & 0 & 33 & 0.07 & 0.25 & 0.10 & 6 & 14 \\
\hline $\mathrm{E}_{4} \mathrm{Bb}_{4} \mathrm{C}_{5}\left(\mathrm{D}_{5}\right) \mathrm{Bb}_{4} \mathrm{Ab}_{4} \mathrm{~F}_{4} \mathrm{G}_{4}$ & 0.83 & 0.77 & 0 & 0 & 0 & 17 & 0 & 33 & 0.78 & 0.63 & 0.27 & 4 & 14 \\
\hline $\mathrm{G}_{4} \mathrm{E}_{4}\left(\mathrm{~F}_{4}\right) \mathrm{C}_{4} \mathrm{~A}_{3} \mathrm{G}_{3} \mathrm{~B}_{3} \mathrm{C}_{4}$ & 0.84 & 0.65 & 0 & 0 & 0 & 0 & 0 & 17 & 1.62 & 1.15 & 1.40 & 13 & 36 \\
\hline $\mathrm{CH}_{4} \mathrm{GH}_{4} \mathrm{CH}_{4} \mathrm{~F}_{4} \mathrm{GH}_{4} \mathrm{C}_{4}\left(\mathrm{Al}_{3}\right) \mathrm{CH}_{4}$ & 0.85 & 0.52 & 0 & 0 & 1 & 50 & 1 & 67 & 1.16 & 0.53 & 0.18 & 3 & 29 \\
\hline $\mathrm{Ab}_{3} \mathrm{C}_{4} \mathrm{Db}_{4}\left(\mathrm{~Eb}_{4}\right) \mathrm{F}_{4} \mathrm{~Eb}_{4} \mathrm{Ab}_{3} \mathrm{C}_{4}$ & 0.87 & 0.65 & 0 & 0 & 0 & 17 & 0 & 33 & 0.66 & 1.07 & 0.02 & 43 & 33 \\
\hline $\mathrm{Bb}_{3} \mathrm{~F}_{4} \mathrm{D}_{4} \mathrm{~A}_{3}\left(\mathrm{G}_{3}\right) \mathrm{Eb}_{4} \mathrm{~F}_{4} \mathrm{Bb} \mathrm{b}_{3}$ & 0.87 & 0.65 & 1 & 17 & 1 & 50 & 1 & 50 & 0.22 & 0.48 & -0.10 & 2 & 14 \\
\hline$G_{4} C_{5} D_{5} B_{4} D_{5}\left(E_{5}\right) F_{4} G_{4}$ & 0.87 & 0.65 & 0 & 0 & 0 & 17 & 1 & 67 & 1.12 & -0.04 & 0.42 & 5 & 17 \\
\hline $\mathrm{F}_{4} \mathrm{~A}_{4} \mathrm{C}_{5} \mathrm{~F}_{5} \mathrm{C}_{5}\left(\mathrm{D}_{5}\right) \mathrm{Bb}_{4} \mathrm{~A}_{4}$ & 0.88 & 0.57 & 0 & 0 & 0 & 0 & 0 & 17 & 1.45 & 0.52 & 1.13 & 17 & 31 \\
\hline$E_{5} A_{4} C_{5} E_{5}\left(F_{5}\right) A_{4} F_{4} A_{4}$ & 0.91 & 0.52 & 0 & 0 & 0 & 33 & 1 & 50 & 0.15 & -0.47 & 0.14 & 4 & 12 \\
\hline $\mathrm{FH}_{4} \mathrm{CH}_{5} \mathrm{~B}_{4} \mathrm{CH}_{5}\left(\mathrm{DH}_{5}\right) \mathrm{G}_{4} \mathrm{FH}_{4} \mathrm{~A}_{4}$ & 0.93 & 0.65 & 0 & 0 & 0 & 17 & 1 & 67 & -0.09 & -0.12 & -0.08 & 10 & 17 \\
\hline $\mathrm{B}_{3} \mathrm{Ft}_{4} \mathrm{G}_{4}^{\#_{4}}\left(\mathrm{G}_{4}\right) \mathrm{E}_{4} \mathrm{~F}_{4} \mathrm{DH}_{4} \mathrm{~B}_{3}$ & 0.94 & 0.65 & 0 & 0 & 0 & 17 & 1 & 50 & 0.96 & 0.99 & 0.46 & 6 & 36 \\
\hline
\end{tabular}

*For the altered melodies in Experiment 2, the note in parentheses replaced the preceding note.

\section{Correlation Between Tonal Structure Ratings and the MKC}

Ratings were averaged separately across subjects in each of the three clusters. Table 1 shows the average normalized ratings from each cluster for each melody. Ratings by Cluster A subjects correlated positively with the MKC values of the melodies $[r(34)=0.72 \dagger]$. To measure the consistency of the three ratings of each melody, correlations were calculated between the first and second ratings, between the second and third ratings, and between the first and third ratings of the melodies. The average of these three correlations is reported as a measure of consistency. Cluster A subjects were the most consistent in their ratings; average consistency was 0.63 . For Cluster B, the correlation with MKC was smaller but also significant $[r(34)=0.40 \dagger]$. These subjects were less consistent in their ratings; average consistency was 0.31 . Average ratings by Cluster $\mathrm{C}$ subjects also correlated significantly with the MKC $[r(34)=0.50 \dagger]$. These subjects were the least consistent in their ratings; average consistency was 0.25 .

For Cluster $\mathrm{C}$ subjects, the first rating of each melody correlated more closely with the MKC $[r(34)=0.61 \dagger]$ than did the second or third ratings of each melody $\left[r(34)=0.30^{*}\right.$ for both the second and third set of ratings]. These subjects may have changed their ratings criteria or response strategy partway through the experiment.

The average ratings by the 3 unusual nonmusician subjects did not correlate with the MKC $[r(34)=0.17$, 0.19 , and 0.10$]$. Two of the 3 unusual subjects were also highly inconsistent in their ratings $(0.16$ and 0.06$)$. They did not display any systematic changes in their ratings across the three repetitions of the melodies. The third unusual nonmusician was highly consistent in his ratings (0.78), which were based on the pitch height of the melodies. Melodies in low pitch registers were rated as high, and ratings systematically decreased as the pitch register of the melodies increased.

\section{Correlation Between Tonal Structure Ratings and Other Measures}

Simplified MKC. The simplified MKC was based on simplified key profiles in which all scale tones received a value of 1 and nonscale tones received a value of $0 .^{3}$ Because the simplified MKC does not differentiate among scale tones, this measure can be compared with the MKC 
to evaluate the need for differential weighting of scale tones within the key profiles.

The simplified MKC produced lower correlations with ratings of tonal structure than did the MKC for all three clusters of subjects $\left[r(34)=0.40^{\dagger}, 0.22\right.$, and $0.34^{*}$ for Clusters $\mathrm{A}, \mathrm{B}$, and $\mathrm{C}$, respectively]. This result suggests that the differentiation of scale tones in the key profiles is an important part of the MKC's success as a predictor of tonal structure ratings. To further test the effect of differentially weighting scale tones, a multiple regression of $\mathrm{MKC}$ and simplified $\mathrm{MKC}$ on the ratings indicated the unique contribution of MKC when simplified MKC was already factored in. This contribution was significant for Clusters A and C, and nearly so for Cluster B $[F(1,33)=18.56 \dagger, 4.08$, and $5.37 \dagger$ for Clusters $\mathrm{A}, \mathrm{B}$, and $C$, respectively].

Temporal measures. Three measures reflecting the temporal ordering of tones in the melodies were evaluated both alone and in combination with the MKC. Fifth span is taken from Longuet-Higgins's (1976, 1978 ) theory of tonality as described by Watkins (1985a, 1985b; Watkins \& Dyson, 1985). According to this theory, each pitch class has a $q$ value within the context of a particular key. The tonal center or tonic pitch has a $q$ value of 0 . The pitch class a perfect fifth ( 7 semitones) above the tonic pitch has a $q$ value of 1 , the pitch class a perfect fifth above that has a $q$ value of 2 , and so on up to a $q$ value of 6 . The pitch class a perfect fifth below the tonic pitch has a $q$ value of -1 , the pitch class a perfect fifth below that has a $q$ value of -2 , and so on down to -5 . Fifth span is the absolute value of the difference in $q$ values between two successive notes. In most melodies, fifth spans greater than 5 are rare, and listeners judged melodies with high fifth spans as less melodious than melodies with low fifth spans when the pitch distributions in the melodies correlated strongly with the key profiles (Watkins, 1985b). This suggests that melodies with low fifth spans should be rated higher in tonal structure than melodies with high fifth spans, particularly for melodies with high MKC values. The fifth-span value for a melody was defined by Watkins as 0 when no fifth spans exceeded 5 and as 1 when one or more fifth spans exceeded 5. Because a continuous measure of fifth span may correlate more strongly with tonal structure ratings than a binary measure, "\%fifth span" represented the percentage of fifth spans greater than 5 .

Semitone span is the number of semitones between two consecutive notes in a melody. In most melodies, large semitone spans are rare, and melodies with large semitone spans are judged as less melodious than melodies with low semitone spans (Watkins, 1985b). Therefore, semitone span should also correlate negatively with tonal structure ratings. Following Watkins (1985b), the binary semitone-span value was 0 for melodies in which the majority of semitone spans was less than 5 semitones (a perfect fourth) and 1 when this was not the case. As a continuous measure, the percentage of semitone spans greater than 5 semitones was represented by "\%semitone span."

Contour represents the complexity of a melody's pitch contour. It is measured by the number of pitch contour changes, that is, changes in pitch direction, either from ascending to descending pitches or from descending to ascending pitches. Cuddy and Lyons (1981) found that melodies with few contour changes received higher ratings of tonal structure than did melodies with many contour changes, except when the melodies included many nonscale pitches, in which case the opposite was true. A binary contour value was coded as 0 for melodies with 2 or fewer contour changes and as 1 for melodies with more than 2 contour changes. As a continuous measure, $\%$ contour represented the number of contour changes in a melody divided by one less than the number of pitches.

Correlations between tonal structure ratings and temporal measures. Of fifth span, semitone span, and contour, only fifth span correlated with tonal structure ratings as well as did the MKC. In combination with the MKC, none of the measures significantly increased the amount of variance in ratings above that accounted for by the MKC alone for all three clusters of subjects. However, for each cluster of subjects, a different temporal measure significantly improved prediction of tonal structure ratings.

Each of the three temporal measures was coded both as a binary variable and as a percentage. The first analysis evaluated which coding was more strongly correlated with tonal structure ratings. For fifth span, binary coding was superior. For Clusters A, B, and C, respectively, $r(34)=-0.73 \dagger,-0.42 \dagger$, and $-0.53 \dagger$ for the binary coding, and $r(34)=-0.48 \dagger,-0.25$, and $-0.35^{*}$ for the percentage coding. For semitone span, percentage coding was superior. For Clusters $\mathrm{A}, \mathrm{B}$, and $\mathrm{C}, r(34)=-0.13$, -0.02 , and -0.11 for the binary coding, and $r(34)=$ $-0.37^{*},-0.31^{*}$, and $-0.46 \dagger$ for the percentage coding. For contour, binary coding was slightly superior. For Clusters $\mathrm{A}, \mathrm{B}$, and $\mathrm{C}, r(34)=-0.34^{*},-0.58 \dagger$, and $-0.28^{*}$ for the binary coding, and $r(34)=-0.25,-0.50 \dagger$, and $-0.29^{*}$ for the percentage coding.

Fifth span, \%semitone span, and contour were then each evaluated in combination with MKC in a multiple regression. Table 2 shows the $R^{2}$ values, which represent the proportion of variance in the ratings accounted for by the two variables. The partial correlation coefficient indicates the amount of variance in the ratings that was not accounted for by MKC but was accounted for by the temporal measure. The partial correlation coefficient for fifth span was significant only for Cluster A. The partial correlation coefficient for \%semitone span was significant only for Cluster $\mathrm{C}$. The partial correlation coefficient for contour was significant only for Cluster B.

The failure of fifth span to consistently improve prediction of tonal structure ratings by the MKC despite the high correlations between fifth span and ratings was due to the significant correlation between MKC and fifth span values $[r(34)=-0.62 \dagger]$. Neither \%semitone span 
Table 2

Correlations Between Tonal Structure and MKC, Fifth Span, \% Semitone Span, and Contour and Multiple Regression on Tonal Structure With MKC and Fifth Span, \% Semitone Span, and Contour

\begin{tabular}{|c|c|c|c|c|c|c|c|c|}
\hline & $\begin{array}{c}\text { Number of } \\
\text { Melodies }\end{array}$ & $r_{\mathrm{MKC}}$ & $\begin{array}{r}r_{\text {Fiffh }} \\
\text { Span }\end{array}$ & $\begin{array}{c}r_{\% \text { Semitone }} \\
\text { Span }\end{array}$ & $r_{\text {Contour }}$ & $\begin{array}{l}R_{\text {MKC \& }}^{2} \\
\quad \text { Fifth Span }\end{array}$ & $\begin{array}{l}R_{\text {MKC \& }}^{2} \\
0 \text { Semitone Span }\end{array}$ & $R_{\text {MKC \& }}^{2}$ \\
\hline Experiment 1 & 36 & & & & & & & \\
\hline Cluster A & & $0.72 \dagger$ & $-0.73 \dagger$ & $-0.37^{*}$ & $-0.34^{*}$ & $0.65 \ddagger$ & 0.56 & 0.59 \\
\hline Cluster B & & $0.40 \dagger$ & $-0.42 \dagger$ & $-0.31^{*}$ & $-0.58 \dagger$ & 0.21 & 0.21 & $0.46 \S$ \\
\hline Cluster C & & $0.50 \dagger$ & $-0.53 \dagger$ & $-0.46 \dagger$ & $-0.28^{*}$ & 0.33 & $0.38 \ddagger$ & 0.31 \\
\hline \multicolumn{9}{|l|}{ Cuddy et al. (1981) } \\
\hline Expt. 1 & 32 & & & & & & & \\
\hline Highly Trained & & $0.70 \dagger$ & $-0.47 \dagger$ & -0.27 & 0.27 & 0.52 & $0.62 \ddagger$ & 0.50 \\
\hline Trained & & $0.50 \dagger$ & $-0.37^{*}$ & $-0.44 \dagger$ & 0.08 & 0.27 & $0.50 \S$ & 0.26 \\
\hline \multicolumn{9}{|l|}{ Cuddy et al. (1981) } \\
\hline Expt. 3 & 20 & & & & & & & \\
\hline Highly Trained & & $0.67 \dagger$ & $-0.40^{*}$ & -0.31 & -0.16 & 0.45 & 0.52 & 0.47 \\
\hline Trained & & $0.57 \dagger$ & -0.36 & -0.32 & -0.19 & 0.33 & 0.42 & 0.37 \\
\hline Untrained & & $0.53 \dagger$ & -0.33 & -0.28 & -0.21 & 0.28 & 0.35 & 0.33 \\
\hline \multicolumn{9}{|c|}{ Cuddy \& Lyons (1981) } \\
\hline Expt. 2 & 10 & $0.83 \dagger$ & $-0.88 \dagger$ & $-0.65^{*}$ & -0.13 & 0.78 & 0.72 & 0.74 \\
\hline Taylor (1976) & 12 & & & & & & & \\
\hline High Ability & & $0.69 \dagger$ & -0.46 & -0.15 & -0.35 & 0.51 & 0.47 & 0.56 \\
\hline Medium Ability & & $0.73 \dagger$ & $-0.54^{*}$ & -0.10 & -0.35 & 0.54 & 0.54 & 0.62 \\
\hline Low Ability & & 0.01 & 0.10 & 0.09 & 0.00 & 0.04 & 0.01 & 0.00 \\
\hline Experiment 2 & 36 & & & & & & & \\
\hline Large Cluster & & $-0.28 *$ & -0.21 & $-0.37^{*}$ & -0.14 & $0.32 \S$ & $0.28 \S$ & 0.11 \\
\hline Small Cluster & & -0.11 & -0.14 & -0.25 & -0.18 & 0.08 & 0.09 & 0.05 \\
\hline
\end{tabular}

${ }^{*} p<.05$ (one-tailed). $\quad \neq p<.01$ (one-tailed). $\ddagger$ Partial correlation coefficient for fifth span, \%semitone span, or contour significant at $p<.05$. §Significant at $p<.01$.

nor contour correlated significantly with the MKC values $[r(34)=-0.23$ and 0.10$]$.

\section{Discussion}

The MKC is an effective measure of the perceived degree of tonal structure in a melody. Both musically trained and untrained subjects generally rated the tonal structure of novel melodies in a manner that correlated positively with the MKC values of the melodies. However, musically untrained subjects tended to be less consistent in their ratings than trained subjects, and this inconsistency was reflected in a somewhat weaker correlation with the MKC.

The importance of functionally differentiating among scale pitches was demonstrated by the superiority of the MKC over the simplified MKC, which weighted pitch classes solely on the basis of scale membership. None of the measures reflecting the temporal ordering of pitches consistently accounted for more of the variance in ratings than was accounted for by the MKC alone. This result may indicate either that the temporal factors do not influence tonal structure ratings or that the particular temporal measures used in this experiment failed to capture the influence of temporal factors on tonal structure ratings.

The greater correlation between the tonal structure ratings and the binary rather than the continuous measures of fifth span and contour suggests that listeners are not sensitive to small differences in these measures. In contrast, listeners do appear to be sensitive to small dif- ferences in the semitone span of melodies when rating tonal structure, as reflected by the stronger correlation between ratings and the continuous rather than the binary measure of semitone span.

The question that arises is: Did the subjects whose tonal structure ratings did not correlate highly with the MKC perceive tonal structure differently from the way in which the majority of subjects did or did their ratings simply reflect alternative response strategies? Evidence from several studies suggests that musically trained and musically untrained listeners perceive tonal structure similarly. For example, Cuddy et al. (1981) found a correlation of $0.95 \dagger(d f=18)$ between tonal structure ratings made by musically trained and musically untrained listeners. Studies of priming effects in musical chord processing (Bharucha, 1987; Bharucha \& Stoeckig, 1986, 1987) also have indicated that listeners with and listeners without musical training represent tonal organization similarly. Developmental studies using the probe-tone rating task to examine the internal key profiles of children (Cuddy \& Badertscher, 1987; Speer \& Meeks, 1985) suggest that, at least by the sixth grade, both musically trained and musically untrained children represent the tonal structure of Western music as adults do.

The low correlation between the MKC and tonal structure ratings by some musically untrained subjects in Experiment 1 is therefore likely to be due not to a difference in their perception of tonal structure, but to a difference in response strategy. The dependence of tonal 
structure ratings by one nonmusician subject on the pitch register of the melodies is an example of an alternative response strategy. Some subjects in probetone experiments (Frankland \& Cohen, 1990; Krumhansl \& Shepard, 1979) similarly based their responses on distance in semitones between the probe tone and the preceding context rather than in terms of the key profile.

\section{MKC ANALYSIS OF OTHER MEASURES OF TONAL STRUCTURE}

In this section, the MKC and the measures reflecting the temporal ordering of tones are evaluated with respect to measures of tonal structure from several other studies.

\section{Cuddy, Cohen, and Mewhort (1981)}

In Experiment 1 of Cuddy et al. (1981), 120 subjects rated 32 seven-note melodies "in terms of [their] tonality or tone structure" (p. 875). Sixty subjects were highly trained musicians who played at least two musical instruments regularly and were accomplished performers. Sixty other subjects had had some informal musical training, and "typically, sang in a choir or were faithful attenders of concert series" (p. 875).

As shown in Table 2, the ratings from both the highly trained and the less trained listeners correlated significantly with the MKC values, and were comparable in size to the correlations in Experiment 1 of the present study. Fifth span did not correlate with tonal structure ratings nearly as well as did the MKC, nor did \%semitone span or contour. Of the temporal measures, only $\%$ semitone span significantly increased the amount of variance accounted for above that accounted for by the MKC, and it did so for both groups of subjects.

In Experiment 3 of Cuddy et al. (1981), highly and moderately trained musicians, as well as a group of relatively untrained subjects who had had less than two years of formal musical training rated the tonal structure of 20 seven-note melodies. The correlation between MKC values of the melodies and ratings were again significant and comparable to those from Experiment 1 . However, when the ratings were correlated with the five levels of harmonic structure into which the authors had categorized the melodies, the correlations were higher $[r(18)=0.93 \dagger, 0.91 \dagger$, and $0.87 \dagger$ for subjects with high, moderate, and low degrees of musical training]. None of the temporal measures correlated with the ratings as strongly as did the MKC, and none significantly increased the amount of variance accounted for in the ratings above what was accounted for by the MKC alone.

\section{Cuddy and Lyons (1981)}

In Experiment 2 of Cuddy and Lyons (1981), six highly trained music students and six professional musicians, all of whom had studied music theory and analysis, rated the structure of 10 seven-note melodies.
The subjects were given the melodies in musical notation and could hum, sing, or play the melodies under no time constraints.

The ratings correlated very highly with the MKC values of the melodies, as shown in Table 2. Again, however, when the ratings were correlated with the five levels of harmonic structure into which Cuddy and Lyons had categorized the melodies, the correlation was higher $[r(8)=0.92 \dagger]$. Fifth span correlated with the ratings as well as did the MKC, perhaps because the MKC and fifth-span values were themselves highly correlated $[r(8)=0.94 \dagger]$. Correlations between the ratings and $\%$ semitone span and contour were smaller. None of the temporal measures in combination with the MKC significantly increased the amount of variance accounted for in the ratings above what was accounted for by the MKC alone.

\section{Taylor (1976)}

Taylor (1976) tested listeners' ability to sing the tonic pitch of 12 different melodies which were 7,11 , or 15 notes long. The 63 subjects were then divided into groups of high, medium, and low ability on the basis of a test of melodic perception ability. Taylor derived tonal strength (TS) values for each melody and each group of subjects on the basis of the degree of consensus among the subjects in a group regarding the tonal center of the melody. A lower TS value indicated greater consensus and thus a greater degree of tonal strength. Because in all of the previous studies, higher values indicated greater degrees of tonal structure, the TS values were multiplied by -1 so that the correlations with the MKC and the other measures would have the same direction as those in the previous studies.

The correlations between the TS values for the highand medium-ability groups and the MKC values of the melodies were significant and comparable to those of the previous studies, as shown in Table 2. For the lowability group, the correlation was not significant. This result may have occurred because subjects with low melodic perception ability either did not understand the concept of a tonal center or had difficulty in accurately singing what they perceived to be the tonal centers. For the low-ability group, none of the temporal measures correlated significantly with TS values, and for the highand medium-ability groups, none correlated more strongly with the TS values than did the MKC. The addition of fifth span, \%semitone span, or contour to MKC did not significantly increase the amount of variance accounted for above that accounted for by MKC alone for any group of subjects.

\section{Summary}

The results of these analyses are similar to those of Experiment 1 . MKC correlated highly with measures of tonal structure from all four experiments (the present Experiment 1; Cuddy et al., 1981; Cuddy \& Lyons, 1981; Taylor, 1976). In contrast, none of the measures reflecting 
the temporal ordering of pitches consistently correlated with tonal structure. In addition, none of the temporal measures combined with the MKC consistently improved predictions of perceived tonal structure above what was predicted by the MKC alone. Again, this result may have occurred either because temporal order does not influence perception of tonal structure or because these particular temporal measures failed to capture this influence.

In two of the studies (Cuddy et al., 1981, Experiment 3, and Cuddy \& Lyons, 1981, Experiment 2), the melodies were classified by the authors into five levels of harmonic structure. These levels correlated more strongly with the ratings than did the MKC. Thus, the harmonic structure analysis appears to better capture listeners' perceptions of tonal structure than does the MKC. However, as a measure of degree of tonal structure, this system is limited by generalizability and subjectivity. The classification system is not readily generalizable beyond the seven-note single-line melodies used in these experiments. Also, the harmonic structure analysis requires a judgment about the harmonic progression implied by the melody, which is quite subjective. This subjectivity was demonstrated in Experiment 1 of Cuddy et al. (1981), in which two professors of music theory and one graduate student in music composition analyzed the harmonic progression implied by each of 32 melodies. On none of the melodies did the three analysts agree exactly, although if asked to simply categorize the melodies into five levels of harmonic structure, they would probably have shown more agreement.

\section{EXPERIMENT 2}

As discussed earlier, several studies have shown that tonal melodies are better remembered than atonal melodies. Because the MKC is a continuous measure of tonal structure, if the memorability of melodies declines monotonically with the perceived degree of tonal structure, then the MKC should make finer predictions of the memorability of melodies than can be made with a simple tonal/atonal dichotomy. Experiment 2 tested listeners' recognition memory of the melodies from Experiment 1 to determine how well the MKC predicted memorability of melodies.

\section{Method}

\section{Subjects}

Twenty-four members of the Johns Hopkins University community participated for pay or as a course requirement. None had participated in Experiment 1. Half of the subjects were musicians, and half were nonmusicians, as defined in Experiment 1.

\section{Stimuli}

The melodies were the same as those used in Experiment 1. In addition, altered versions of each test melody and practice melody were constructed. As shown in Table 1, one note was changed by either one or two semitones in the altered versions. The alterations did not change the pitch contour of the melodies.

Melodies with more pitch contour changes are more difficult to remember than melodies with fewer contour changes (Boltz,
Marshburn, Jones, \& Johnson, 1985; Cuddy et al., 1981; Cuddy \& Lyons, 1981; Morrongiello, Trehub, Thorpe, \& Capodilupo, 1985). Number of contour changes was therefore controlled with respect to the MKC. When the test melodies were divided into two groups of 18 melodies each on the basis of their MKC values, melodies in the low MKC group had an average of 2.67 pitch contour changes, and melodies in the high MKC group had an average of 2.72 contour changes.

Because larger differences in MKC values between a melody and its alteration may be easier to detect than smaller differences, alterations were chosen to minimize the change in MKC value. The change in MKC value was also controlled with respect to the MKC values of the melodies. Alterations changed the MKC value by an average of .08 in the low MKC group of melodies and by .07 in the high MKC group.

Finally, because detectability of pitch changes in seven- or eight-note melodies is subject to both recency and primacy effects (Divenyi \& Hirsh, 1978; Greene \& Samuel, 1986; Williams, 1975), the serial position of the altered note was controlled with respect to the MKC. The altered note never occurred in the first or last serial position, and the average serial position of the altered note was 3.83 for both the high and the low MKC groups of melodies.

\section{Procedure}

Subjects were read instructions stating that in each trial, they would first hear a standard melody, and that, after a brief pause, they would hear two comparison melodies, one identical to the standard melody and the other slightly altered. Their task was to indicate whether the first or the second comparison melody was identical to the standard melody. The subjects marked their responses on an answer sheet that was covered by a colored sheet of paper so that they could not see their responses to previous trials.

On each trial, a trial number was announced, followed by a standard melody. After a 2.25 -sec silence, the two comparison melodies, separated by $0.5 \mathrm{sec}$ of silence, were presented. The subjects had $4 \mathrm{sec}$ in which to respond before the next trial number was announced.

The subjects first completed a practice block of 12 trials and were told the correct answer after each trial. Data from the practice block were not included in the analysis. Each of the 36 melodies was then tested six times. For each melody, the altered version was presented as the first comparison melody on half of the trials and as the second comparison melody on the other half. Melodies were presented in random order without replacement in six blocks of 36 trials each.

The subjects were tested in a quiet room in groups of up to 4 . After completing all trials, which took about $1 \mathrm{~h}$, the subjects answered a short questionnaire about their musical experience.

\section{Results}

The correlations between the MKC values of the melodies and percentages of errors made on the melodies were quite small, although more errors were made on the melodies with the lowest MKC values than on the melodies with the highest MKC values. Performance was more highly correlated with the nature of the altered note than with the MKC values of the melodies, and did not correlate with the tonal structure ratings from Experiment 1.

\section{Cluster Analysis}

For each subject, the percentage of errors was counted separately for each melody. A cluster analysis was then performed to determine groups of subjects who performed similarly on the task. The percentage of errors 
each subject made on each melody was correlated with the percentage of errors made by every other subject on the same melodies. The correlations were subtracted from 1 to derive a distance matrix. The distance matrix was analyzed as in the previous experiment, by a hierarchical clustering procedure using the centroid method.

There were a large cluster of 16 subjects, including 10 musicians with an average of 7.0 years of private music lessons $(S D=1.9)$ and 6 nonmusicians; a small cluster of 7 subjects, including 2 musicians, with 9 and 7 years of music lessons, and 5 nonmusicians; and 1 unusual nonmusician. Musicians were significantly more likely to be in the large cluster than in the small cluster or to be outliers $\left[\chi^{2}(1, N=24)=5.33^{*}\right]$. The two clusters were distinguished largely on the basis of overall performance. Subjects in the large cluster made an average of $12.9 \%$ errors, and subjects in the small cluster made an average of $26.7 \%$ errors. The percentage of errors made by the large and small clusters on each melody is included in Table 1.

\section{Correlations Between Error Percentages and the MKC}

The correlations between the MKC and error percentages were small and significant only for the large cluster of subjects $\left[r(34)=-0.28^{*},-0.11\right.$, and 0.04 for the large cluster, small cluster, and unusual subject]. The negative correlations indicate that more errors were made on melodies with low MKC values, as expected. As shown in Table 2, correlations between error percentages and each of the temporal measures were also small, and their negative direction was unexpected. Because the temporal measures generally correlated negatively with tonal structure ratings, they were expected to correlate positively with error percentages. Although, for the large cluster, both fifth span and \%semitone span significantly increased the amount of variance accounted for above that accounted for by the MKC alone, the total percentage of variance accounted for $\left(R^{2}\right)$ never exceeded 0.32 .

The weak correlation between MKC and error percentages at first appears inconsistent with reports that atonal melodies are less well remembered than tonal melodies (Cohen, 1982; Dewar et al., 1977; Frances, 1958/1988; Watkins, 1985b). However, in these experiments, stimulus melodies were either highly tonal or highly atonal. To form a comparable test, the eight melodies with the highest MKC values $(0.85-0.94)$ were compared with the eight melodies with the lowest MKC values $(0.35-0.41)$. A repeated-measures general linear models (GLM) analysis, in which cluster membership was a between-subject variable and the MKC value of the melodies was a binary within-subject variable, showed a main effect of MKC value $[F(1,21)=35.91 \dagger]$. More errors were made on melodies with the lowest MKC values than on melodies with the highest MKC values. A significant interaction between cluster membership and MKC values $[F(1,21)=9.60 \dagger]$ reflected that the effect of MKC value was greater for subjects in the large cluster than for those in the small cluster. Subjects in the large cluster made $11.3 \%$ errors on melodies with the highest MKC values and $21.6 \%$ errors on melodies with the lowest MKC values. In contrast, subjects in the small cluster made $23.5 \%$ and $26.7 \%$ on melodies with the highest and lowest MKC values, respectively. The results of Experiment 2 are therefore not inconsistent with reports that tonal melodies are better remembered than atonal melodies.

Informal comments from subjects suggested that when the altered note was the highest or the lowest pitch in the melody, the change was easy to detect. The correlations between this variable and error percentages were significant $[r(34)=0.42 \dagger$ and $0.63 \dagger$ for the large and small cluster] and were greater than those between the MKC and error percentages, particularly for the small cluster.

The correlation between error percentages and tonal structure ratings from Experiment 1 was also calculated. Cuddy et al. (1981) reported correlations ranging from $0.81 \dagger$ to $0.90 \dagger(d f=18)$ between ratings of tonal structure and performance in a melody-recognition task. However, in the present experiment, error percentages by neither cluster of subjects correlated significantly with tonal structure ratings made by any cluster of subjects from Experiment 1 [ $r(34)$ ranged from -0.11 to 0.22 ].

\section{Discussion}

The results of Experiment 2 suggest that the MKC does not make finer predictions of the memorability of melodies than does a binary measure of tonal structure. However, the MKC can be used to identify melodies with very low and very high degrees of tonal structure. The limited success of the MKC in predicting memory performance contrasts with its much greater success in predicting tonal structure ratings. At least two factors may have contributed to this difference: the different types of cognitive processing required by the two tasks and the opportunity for factors other than tonal structure to affect the memory task.

The memory task required detection of a single changed pitch. Altered patterns preserved the pitch contour of the melodies, and changes to the MKC values were minimal. Subjects therefore could not detect the altered melody by comparing pitch contour or tonal structure, which are global properties of the melodies. Rather, the task required processing of the successive pitch intervals in the melodies. In contrast, the tonal-structure rating task required a judgment about an entire melody because tonal structure is a property of a set of notes and not of individual notes or intervals. Because the MKC reflects a global property of melodies, it is perhaps not surprising that direct ratings of tonal structure more closely corresponded to MKC values than did memory performance.

Second, although the rating task directly measured perceived degree of tonal structure, the memory task only indirectly reflected perceived tonal structure. Moreover, this indirect measure was complicated by the pos- 
sibility that factors other than tonal structure could affect the memorability of melodies. Although several factors that may influence the memorability of melodies were controlled in this experiment, other uncontrolled factors may have affected performance. The finding that subjects were better at detecting an altered melody when the altered note was the highest or lowest pitch in the melody is one example.

In the present experiments, performance on the memory task did not correlate with ratings of tonal structure, unlike the studies of Cuddy et al. (1981), who found strong correlations between memory performance and ratings. The difference may have arisen because in the present memory experiment incorrect and correct comparison melodies were roughly equated in MKC values. In contrast, in Cuddy et al.'s experiment, the altered note in the incorrect comparison melodies was always outside the key of diatonic melodies, and thus the degree of tonal structure differed in the correct and incorrect comparison melodies. For melodies with high degrees of tonal structure, listeners could therefore detect incorrect melodies by the change in degree of tonal structure in Cuddy's experiment but not in the present experiment.

It is unlikely that the weak correlation between the MKC and memory performance occurred because listeners had insufficient time to interpret the tonal structure of the melodies. The same melodies were used in Experiments 1 and 2, and in Experiment 1, subjects made tonal structure ratings in a limited amount of time, and these ratings were consistent and correlated strongly with the MKC. Other experiments have shown that listeners perceive the tonal center of musical samples after hearing only a few notes (Cohen, 1991), and no more information about the tonal center is gained after five or six notes of nonmodulating melodies (Howell, West, \& Cross, 1984; Taylor, 1976). However, the brief retention interval in Experiment 2 may have worked against an influence of tonal structure on melody recognition. Dowling (1991; Dowling \& Bartlett, 1981) found that over long retention intervals, tonal melodies are less likely to be encoded in terms of pitch contour and more likely to be encoded relative to a tonal center.

An additional possibility is that different degrees of tonal structure affected memory performance indirectly by mapping onto a binary processing strategy in the memory task. Performance was determined by the effectiveness of the processing strategy rather than directly by the tonal structure of the melodies. For example, if the degree of tonal structure of a melody exceeded some threshold level, then a listener might have encoded the pitches of the melody with respect to the tonal center of the melody. However, if the tonal structure was below the threshold level, then, because the tonal center was less apparent, the listener might have used a less successful strategy such as encoding only the direction and approximate size of the pitch interval between successive notes. If performance did not vary substantially within a strategy, this would result in a large difference between highly tonal and highly atonal melodies but only a weak correlation between performance and tonal structure, as was the case in Experiment 2. Several other studies have reported a similar pattern. Cuddy and Lyons (1981) found better recognition of melodies at the highest level of harmonic structure than of melodies at the lower four levels, which did not differ among themselves. Long (1977) tested listeners' abilities to determine whether a probe tone presented after a melody had been included in the melody. Subjects were significantly more accurate with tonal than with atonal melodies. However, the correlation between performance and a continuous measure of tonal strength based on the degree of consensus among listeners as to the tonal center of the melodies (Taylor, 1976) was not significant $[r(34)=-0.22]$.

\section{GENERAL DISCUSSION}

The interpretation of the MKC as a measure of the degree of tonal structure in a melody appears to be valid, as shown by the consistently high correlations between the MKC and various measures of perceived tonal structure. The MKC did not successfully predict performance on a memory task. However, it could identify highly tonal melodies, which were accurately recognized, and highly atonal melodies, which were not accurately recognized. Its ease of use makes the MKC a convenient tool for describing the degree of tonal structure.

The MKC is presumably also applicable to music based on other tonal systems. Key profiles, that is, measurements of the stabilities of pitch classes within various tonal contexts, have been empirically determined for Indian (Castellano, Bharucha, \& Krumhansl, 1984), Balinese (Kessler, Hansen, \& Shepard, 1984), and serial music (Krumhansl, Sandell, \& Sergeant, 1987). From these key profiles, the MKC can be calculated to determine the degree to which a musical sample instantiates a particular tonal system.

As a measure of tonal structure, the MKC provides a descriptive tool for the stimuli used in many studies that examine the effects of tonal structure (e.g., Cohen, 1982; Croonen \& Kop, 1989; Dewar et al., 1977; Dowling, 1990, 1991; Lynch et al., 1991; Umemoto et al., 1989). These stimuli are generally brief single-line melodies, such as were used in these experiments. The MKC makes it possible to objectively describe the tonal structure of musical stimuli and to compare the results of different experiments involving tonal structure as a variable. However, the MKC has not been evaluated as a measure of the tonal structure of larger musical samples, and may not be as effective with such samples. Music is a temporal experience, and the perception of tonal structure may vary over the course of a composition. The MKC calculated over an entire composition may not reflect the tonal structure of the composition as accurately as MKC values calculated over limited portions of a composition reflect the tonal structure of those portions.

In line with Krumhansl's (1990) suggestion that the key-finding algorithm might be improved by the inclusion of temporal, rhythmic, and harmonic factors, the 
MKC may be similarly improved. Although attempts to incorporate the temporal ordering of tones by measures of fifth span, semitone span, and pitch contour complexity were unsuccessful in improving the prediction of tonal structure ratings or memory performance, other measures may be more successful. In samples with a strong rhythmic structure, weighting tones according to their temporal locations within a metrical framework (cf. Palmer \& Krumhansl, 1990; Takeuchi, 1993) may improve the measure of tonal structure. Taking into account the harmonic relations between simultaneously sounded notes in harmonic and polyphonic applications may also improve the measure. Because analyses of tonal structure based on the harmonic progression implied by a single-line melody (Cuddy et al., 1981; Cuddy \& Lyons, 1981) correlated more strongly with tonal structure ratings than did the $\mathrm{MKC}$, the inclusion of harmonic progression is also likely to improve the measurement of tonal structure. To apply harmonic analysis to single-line melodies, however, would require the development of an objective means of determining the harmonic progression underlying a melody, which is a far from trivial problem.

\section{REFERENCES}

BHARUCHA, J. J. (1987). Music cognition and perceptual facilitation: A connectionist framework. Music Perceptia , 5, 1-30.

Bharucha, J. J., \& StoeCKIG, K. (1986). Reaction time and musical expectancy: Priming of chords. Journal of Experimental Psychology: Human Perception \& Performance, 12, 403-410.

Bharucha, J. J., \& Stoeckig, K. (1987). Priming of chords: Spreading activation or overlapping frequency spectra? Perception \& Psychophysics, 41, 519-524.

Boltz, M., Marshburn, E., Jones, M. R., \& Johnson, W. W. (1985). Serial-pattern structure and temporal-order recognition. Perception \& Psychophysics, 37, 209-217.

Brown, H. (1987). Tonal hierarchies and perceptual context: An experimental study of music behavior. Psychomusicology, 7, 77-90.

Brown, H. (1988). The interplay of set content and temporal context in a functional theory of tonality perception. Music Perception, 5, 219-250.

BUTLER, D. (1990). A study of event hierarchies in tonal and post-tonal music. Psychology of Music, 18, 4-17.

ButLER, D., \& BROWN, H. (1984). Tonal structure versus function: Studies of the recognition of harmonic motion. Music Perception, 2, 6-24.

Castellano, M. A., Bharucha, J. J., \& Krumhansl, C. L. (1984). Tonal hierarchies in the music of North India. Journal of Experimental Psychology: General, 113, 394-412.

CoHEN, A. J. (1982). Exploring the sensitivity to structure in music. Canadian University Music Review, 3, 15-30.

CoHen, A. J. (1991). Tonality and perception: Musical scales primed by excerpts from The Well Tempered Clavier of J. S. Bach. Psychological Research, 53, 305-314.

Croonen, W. L. M., \& KoP, P. F. M. (1989). Tonality, tonal scheme, and contour in delayed recognition of tone sequences. Music Perception, 7, 49-68.

Cuddy, L. L., \& Badertscher, B. (1987). Recovery of the tonal hierarchy: Some comparisons across age and levels of musical experience. Perception \& Psychophysics, 41, 609-620.

Cuddy, L. L., Cohen, A. J., \& Mewhort, D. J. K. (1981). Perception of structure in short melodic sequences. Journal of Experimental Psychology: Human Perception \& Performance, 7, 869-883.

Cuddy, L. L., \& Lyons, H. I. (1981). Musical pattern recognition: A comparison of listening to and studying tonal structures and tonal ambiguities. Psychomusicology, 1, 15-33.
Dewar, K. M., Cuddy, L. L., \& Mewhort, D. J. K. (1977). Recognition memory for single tones with and without context. Journal of Experimental Psychology: Human Learning \& Memory, 3, 60-67.

DivenYI, P. L., \& HiRSH, I. J. (1978). Some figural properties of auditory patterns. Journal of the Acoustical Society of America, 64, 1369-1385.

Dowling, W. J. (1990). Expectancy and attention in melody perception. Psychomusicology, 9, 148-160.

Dowling, W. J. (1991). Tonal strength and melody recognition. Perception \& Psychophysics, 50, 305-313.

Dowling, W. J., \& BARTLETT, J. C. (1981). The importance of interval information in long-term memory for melodies. Psychomusicology, 1, 30-49.

FRANCES, R. (1988). The perception of music (W. J. Dowling, Trans.). Hillsdale, NJ: Erlbaum. (Original work published 1958)

Frankland, B., \& CohEN, A. J. (1990). Expectancy profiles generated by major scales: Group differences in ratings and reaction time. Psychomusicology, 9, 173-192.

GREene, R., \& SAMUel, A. (1986). Recency and suffix effects in serial recall of musical stimuli. Journal of Experimental Psychology: Learning, Memory, \& Cognition, 12, 517-524.

Howell, P., WeST, R., \& CROSS, I. (1984). The detection of notes incompatible with scalar structure. Journal of the Acoustical Society of America, 76, 1682-1689.

Janata, P., \& Reisberg, D. (1988). Response time measures as a means of exploring tonal hierarchies. Music Perception, 6, 161-172.

Kessler, E. J., Hansen, C., \& ShePard, R. N. (1984). Tonal schemata in the perception of music in Bali and the West. Music Perception, 2, 131-165.

KrumhansL, C. L. (1979). The psychological representation of musical pitch in a tonal context. Cognitive Psychology, 11, 346-374.

KrumhansL, C. L. (1990). Cognitive foundations of musical pitch. New York: Oxford University Press.

Krumhansl, C. L., \& Kessler, E. J. (1982). Tracing the dynamic changes in perceived tonal organization in a spatial representation of musical keys. Psychological Review, 89, 334-368.

Krumhansl, C. L., Sandell, G. J., \& Sergeant, D. C. (1987). The perception of tone hierarchies and mirror forms in twelve-tone serial music. Music Perception, 5, 31-78.

Krumhansl, C. L., \& ShePard, R. N. (1979). Quantification of the hierarchy of tonal functions within a diatonic context. Journal of Experimental Psychology: Human Perception \& Performance, 5, 579594.

LoNG, P. A. (1977). Relationship between pitch memory in short melodies and selected factors. Journal of Research in Music Education, 25, 272-282.

Longuet-Higgins, H. C. (1976). Perception of melodies. Nature, 263, 646-653.

Longuet-Higgins, H. C. (1978). The perception of music. Interdisciplinary Science Reviews, 3, 148-156.

Lynch, M. P., Eilers, R. E., Oller, D. K., Urbano, R. C., \& WilSON, P. (1991). Influences of acculturation and musical sophistication on perception of musical interval patterns. Journal of Experimental Psychology: Human Perception \& Performance, 17, 967-975.

Morrongiello, B. A., Trehub, S. E., Thorpe, L. A., \& CapoDILUPO, S. (1985). Children's perception of melodies: The role of contour, frequency and rate of presentation. Journal of Experimental Child Psychology, 40, 279-292.

Palmer, C., \& Krumhansl, C. L. (1990). Mental representations of musical meter. Journal of Experimental Psychology: Human Perception \& Performance, 16, 728-741.

ParncutT, R. (1989). Harmony: A psychoacoustical approach. New York: Springer-Verlag.

SOKAL, R. R., \& MiCHENER, C. D. (1958). A statistical method for evaluating systematic relationships. University of Kansas Science Bulletin, 38, 1409-1438.

SPEER, J. R., \& MEeKs, P. U. (1985). School children's perception of pitch in music. Psychomusicology, 5, 49-56.

TAKeUCHI, A. H. (1993, May). Perceptual organization of temporal patterns in music. Paper presented at meeting of the Acoustical Society of America, Ottawa. 
TAYLOR, J. A. (1976). Perception of tonality in short melodies. Journal of Research in Music Education, 24, 197-208.

Umemoto, T., Mikumo, M., \& Murase, A. (1989). Development of tonal sense-a new test on cognition of pitch deviation. Human Developmental Research, 5, 155-174.

WATKINS, A. J. (1985a). Perceptual aspects of synthesized approximations to melody. Journal of the Acoustical Society of America, 78, $1177-1186$

WATKINS, A. J. (1985b). Scale, key, and contour in the discrimination of tuned and mistuned approximations to melody. Perception \& Psychophysics, 37, 275-285.

Watkins, A. J., \& Dyson, M. C. (1985). On the perceptual organisation of tone sequences and melodies. In P. Howell, I. Cross, \& R. West (Eds.), Musical structure and cognition (pp. 71-119). London: Academic Press.

WILLIAMS, D. B. (1975). Short-term retention of pitch sequence. Journal of Research in Music Education, 23, 53-66.

\section{NOTES}

1. The term atonal is used in this article to describe a musical sample that lacks tonal structure in the sense of having a clear tonal cen- ter that governs the relative importance of pitches. In this context, atonal does not refer to the particular style of 20th-century music in which establishment of a particular pitch as a tonal center was specifically avoided.

2. Significance is indicated by * at the $p<.05$ level and by $\dagger$ at the $p<.01$ level. A one-tailed significance test was used for all correlations.

3. In the simplified MKC, pitch classes that were both a minor seventh and a major seventh above the tonic pitch were counted as scale tones in minor keys because, in Krumhansl and Kessler's (1982) minor-key profile, both of these pitch classes received ratings comparable to those of the other scale tones. The pitch class a minor sixth above the tonic pitch was counted as a scale tone, but the pitch class a major sixth above the tonic pitch was not, also in accordance with the minor-key profile.
(Manuscript received April 16, 1993; revision accepted for publication March 25, 1994.) 